\title{
Revisão Sistemática de Pesquisas sobre Eficácia Coletiva Docente
}

\author{
Maély Ferreira Holanda Ramos \\ Universidade Federal do Pará (UFPA) \\ Fernando Augusto Ramos Pontes \\ Universidade Federal do Pará (UFPA) \\ Simone Souza Costa e Silva \\ Universidade Federal do Pará (UFPA) \\ Ana Patrícia de Oliveira Fernandez \\ Instituto Federal do Pará (IFPA)
}

Karla Cristina Nina

Instituto Federal do Pará (IFPA)

\begin{abstract}
RESUMO
A eficácia coletiva docente é a percepção compartilhada pelos professores sobre a capacidade do corpo docentede organizar e executar ações necessárias a um efeito positivo no desempenho acadêmico dos alunos. Este artigo tem o objetivo de construir um panorama das pesquisas sobre eficácia coletiva de professores, analisando seus principais resultados. Para tanto, foram realizadas buscas de artigos publicados entre 2000 e 2013, na base de dados Portal de Periódicos Capes. Os resultados indicam que a eficácia coletiva de professores está diretamente relacionada ao desempenho acadêmico dos alunos. Notou-se ainda que outras variáveis podem influenciar as crenças coletivas, tais como a autoeficácia e a situação socioeconômicada escola.
\end{abstract}

Palavras-chave: crenças de eficácia coletiva docente, revisão sistemática, contexto escolar.

\begin{abstract}
Systematic Review on Researches about Collective Teacher Efficacy

The collective teacherefficacy is the shared perception of teachers about the capability of high school teachers to organize and execute necessary actions, in order to have a positive effect on the academic performance of students. This paper aims to show an overview research on collective efficacy of teachers, analyzing its main results. Researches for articles published from 2000 to 2013 on the databases of CAPES Journals were performed. The results indicate that collective teacher efficacy is directly related to student achievement. It was also noted that other variables may influence on the collective beliefs, such as self-efficacy and the socioeconomic status of the school.
\end{abstract}

Keywords: collective efficacy beliefs in teaching, systematic review, school context.

A contribuição das organizações escolares, suas dinâmicas e principais características para o sucesso acadêmico de seus alunos têm sido objeto de interesse de vários pesquisadores (Bandura, 1986, 1993; Bandura, Lindzey, \& Runyan, 2007; Klassen, Tze, Betts, \& Gordon, 2010; Kurz \& Knight, 2004; Tschannen-Moran, Hoy, W. \& Hoy, 1998). Uma característica institucional que tem sido relacionada com o desempenho acadêmico dos alunos é a eficácia coletiva docente, assim como a autoefácia e a satisfação no trabalho (Bandura, 1993, 1997).

A eficácia coletiva docente é a percepção compartilhada pelos professores de uma escola sobre a capaci- dade do corpo docente, como um todo, para organizar e executar ações necessárias a um efeito positivo sobre o desempenho acadêmico dos alunos(Stephanou, 2013).

Com base na Teoria Social Cognitiva de Bandura (1977, 1986, 1997), esse constructo está relacionado ao modo como as pessoas exercem controle sobre as próprias vidas, ou às crenças nas próprias capacidades para produzir determinada ação. A Teoria Social Cognitiva considera as crenças de eficácia como as bases da agência humana, que operam dentro de múltiplas influências socioestruturais (Bandura, 1997).

A eficácia coletiva docente, numa organização social, constitui-se como produto da interação dinâmica en- 
tre os professores, sendo uma importante propriedade escolar, haja vista a associação positiva com os resultados acadêmicos (Bandura, 1993, 1997). Tal noção não se restringe à soma das crenças individuais de cada componente do grupo, mas, sim, está baseada na compreensão de que as crenças partilhadas pelo coletivo podem favorecer resultados esperados (Dantas, Guerreiro-Casanova \& Azzi, 2000), sendo, portanto, uma propriedade emergente em nível grupal(Bandura, 2000).

Segundo a Teoria Social Cognitiva, o lócus das crenças de eficácia coletiva reside na mente dos componentes do grupo, que age através do comportamento de seus membros. Assim, os indivíduos do grupo atuam de forma coordenada, orientados por uma crença comum, compartilhada por todos, agindo na manutenção e no aumento da motivação para o cumprimento das funções (Bandura, 2000; Bzuneck \& Guimarães, 2009). Considera-se que a eficácia coletiva influencia o funcionamento de grupos em sistemas sociais diversos, incluindo organizações empresariais, equipes esportivas, equipes de combate, grupos de ação política e sistemas educacionais (Bandura, 2000).

Alguns elementos do contexto escolar podem contribuir para o desenvolvimento da eficácia coletiva de professores, de modo que são considerados fontes de informações importantes para a construção dessas crenças.

Bandura $(1996,1997)$ postulou quatro fontes de informação sobre as crenças de eficácia: experiência direta, experiência vicária, persuasão verbal e estados afetivos e fisiológicos. O modo como tais fontes são interpretadas é fundamental, pois as mesmas experiências podem resultar em crenças de eficácia diferentes, dependendo da interpretação realizada pela pessoa (Bandura, 1992, 1997).

A primeira fonte é a experiência direta, que é caracterizada pelas experiências interpretadas como bemsucedidas. Essas percepções geralmente aumentam a confiança; experiências interpretadas como mal sucedidas comumente rebaixam a confiança (Goddard, Hoy, W. \& Hoy, 2000). A segunda fonte denomina-se experiência vicária e está relacionada ao que aprendemos por meio das experiências dos outros. A observação de vivências de sucesso pode elevar as crenças (Bandura, 1994).

A terceira fonte é a persuasão social e envolve contribuições verbais de outros, como colegas, supervisores e administradores, servindo para fortalecer as crenças principalmente quando há a necessidade de en- frentamento de desafios e superação de dificuldades (Tschannen-Moran \& McMaster, 2013). Finalmente, os estados afetivos e fisiológicos também são fontes de informação da eficácia coletiva docente(Goddard, 2001). Segundo Bandura (1997), ao julgar suas próprias capacidades, as pessoas confiam parcialmente em informações transmitidas por estados fisiológicos e emocionais.

É importante enfatizar que as fontes de eficácia indicadas por Bandura (1986) ajudam a construir tanto as crenças individuais (autoeficácia) quanto as crenças coletivas. Enquanto a eficácia coletiva docente se refere às crenças que os professores têm na capacidade do grupo ao qual pertencem, a autoeficácia se refere às crenças dos indivíduos em suas próprias capacidades para realizar com sucesso um curso particular de ação, visando ao alcance de objetivos estabelecidos.

Diante do contexto apresentado, o presente estudo analisa 30 artigos levantados na base de dados Portal de Periódicos Capes, com vistas a investigar seus principais resultados para o aprofundamento da compreensão da eficácia coletiva docente. Para tanto, analisam-se as relações entre a eficácia coletiva e outras variáveis emergentes do contexto escolar.

\section{Estratégias de busca e análise}

Para realizar o presente levantamento, foram definidos alguns descritores relacionados à temática em questão, os quais geraram 36 possibilidades de buscas a partir de seus cruzamentos, a saber: collective efficacy, collective efficacy beliefs, teacher collective efficacy, collective perceptions, social cognitivetheory, teacher efficacy. Ressalta-se que os respectivos descritores foram aplicados às buscas também em português para levantamento de estudos brasileiros.

Visando ao delineamento da pesquisa, foram pré-determinados alguns critérios de inclusão, sendo estes: conter no título o termo collective efficacy ou eficácia coletiva; estar completo e disponível; apenas artigos dos últimos 13 anos (2000 a 2013); artigos em inglês e português; ter sido revisado por pares; e ter como foco investigar o constructo da eficácia coletiva de professores a partir da Teoria Social Cognitiva. Todos os achados que não obedeceram aos critérios de inclusão mencionados foram excluídos.

Foram encontrados nas buscas apenas 30 artigos que atendiam a todos os critérios de inclusão. Foi possível localizar $70 \%$ dos artigos a partir do descritor collective efficacy. O restante dos descritores totalizou percentuais menores que $7 \%$. Para análise dos dados, organizaramse os resultados em planilhas no Microsoft Excel (2010). 
Os dados foram organizados considerando-se os principais resultados e estruturados em categorias temáticas, a saber: (a) autoeficácia de professores; (b) desempenho de alunos; (c) situação socioeconômica; (d) satisfação no trabalho; (e) tempo de serviço; e (f) estresse no trabalho. As categorias temáticas foram formadas a partir das variáveis que mais se relacionaram com a eficácia coletiva docente.

Ressalta-se que, por essas não serem categorias exclusivas, um mesmo artigo pode ter investigado mais de uma temática. Sendo assim, a soma dos percentuais de incidência indicada na apresentação dos resultados, ultrapassou o total de $100 \%$ dos artigos analisados.

$\mathrm{Na}$ análise dos dados, utilizaram-se as abordagens qualitativa e quantitativa. $\mathrm{Na}$ abordagem quantitativa, buscou-se comparar percentuais e fazer a distribuição de frequência dos dados. Em alguns casos, os resultados foram demonstrados por meio de representação numérica em tabela (Cozby, 2003).

$\mathrm{Na}$ abordagem qualitativa, tomou-se por base a análise de conteúdo de Bardin (1975). Esse é um método frequentemente adotado em pesquisas qualitativas, embora também seja utilizado em pesquisas quantitativas. A análise de conteúdo envolve iniciativas de explicitação, sistematização e expressão do conteúdo de mensagens.

Assim, transitou-se entre dois polos na investigação científica: o rigor da objetividade e a fecundidade da subjetividade. Esses fatores implicaram a definição e o tratamento dos indicadores qualitativos e quantitativos (Minayo \& Sanches, 1993).

\section{RESULTADOS E DISCUSSÃO}

A partir da análise dos resultados, foi possível identificar as principais variáveis que mais têm sido relacionadas à eficácia coletiva docente. Assim, construiu-se o mapa das relações desse constructo, formando um panorama dos estudos sobre a temática nos últimos anos. As principais variáveis são: (1) desempenho de alunos; (2) autoeficácia de professores; (3) situação socioeconômica; (4) satisfação no trabalho; (5) tempo de serviço; e (6) estresse no trabalho. Na Tabela 1, é possível visualizar os percentuais e as frequências de incidência dessas variáveis.

\section{Tabela 1}

Frequências e Percentuais das Principais Variáveis

\begin{tabular}{lcc}
\hline \multicolumn{1}{c}{ Variáveis } & Frequência & Percentuais \\
\hline Autoeficácia de professores & 11 & $36 \%$ \\
Desempenho de alunos & 11 & $36 \%$ \\
Situação Socioeconômica (SES) & 6 & $20 \%$ \\
Satisfação no trabalho & 2 & $6 \%$ \\
Tempo de serviço & 2 & $6 \%$ \\
Estresse no trabalho & 2 & $6 \%$ \\
\hline
\end{tabular}

Ressalta-se que essas variáveis foram identificadas a partir da maior frequência conjunta com a eficácia coletiva docente. Sendo assim, focalizam-se as análises nas relações entre as principais variáveis identificadas nos resultados dos artigos (Figura 1). As relações exploradas nesta revisão sistemática direcionam-se para os delineamentos correlacionais e para a causalidade entre as variáveis, pois essas são as análises mais frequentemente realizadas nas pesquisas.

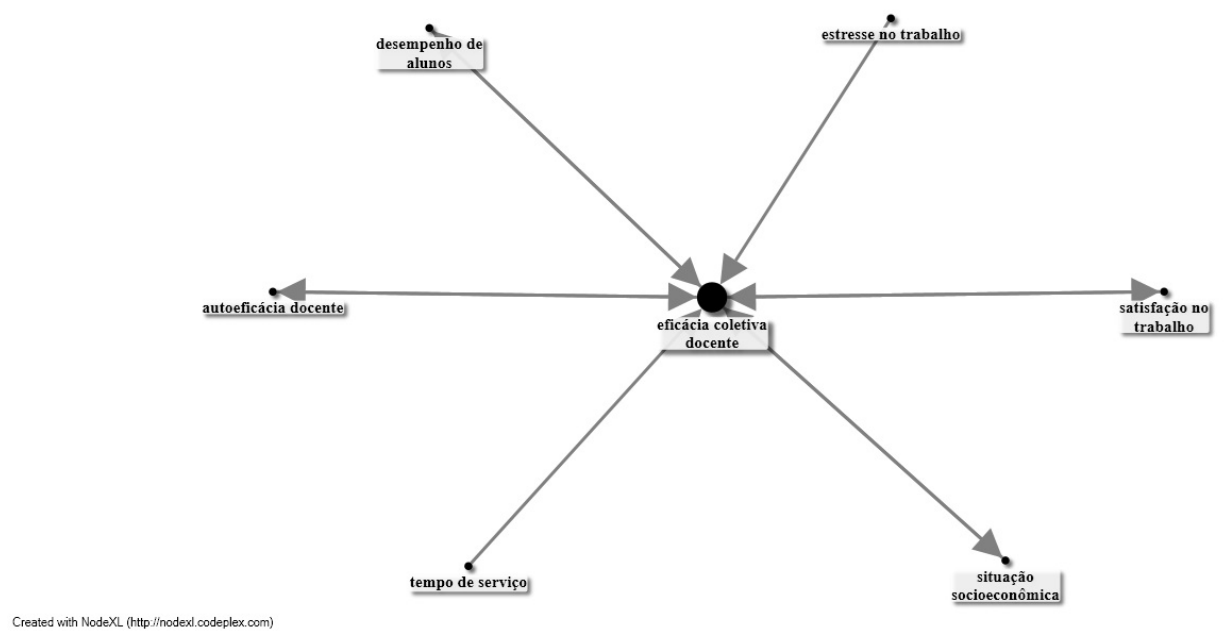

Figura 1. Mapa conceitual dos estudos sobre a eficácia coletiva docente 
Com os delineamentos correlacionais, apenas se observam e se registram as mudanças nas variáveis, tentando perceber o nível de covariação entre elas e identificando a forma como covariam e se isso faz sentido. Deve-se entender, no entanto, que não se pode inferir uma causação somente a partir de uma correlação, pois a relação entre as variáveis não informa, significativamente, muita coisa sobre causa e efeito, apenas indica uma direção (Dancey \& Reidy, 2006).

A figura 1 consiste num mapa conceitual que resume as relações entre as principais variáveis investigadas nesta revisão sistemática. Apresenta-se a direção das relações por meio de setas que ligam as variáveis. Nota-se que, em síntese, a eficácia coletiva de professores é influenciada pelo estresse no trabalho e pelo tempo de serviço. O mapa conceitual indica também que há uma influência mútua entre eficácia coletiva docente e as seguintes variáveis: desempenho de alunos, autoeficácia, situação socioeconômica e satisfação no trabalho.

\section{Autoeficácia de professores}

A variável autoeficácia de professores totalizou $36 \%$ dos estudos levantados. Os resultados indicaram que $100 \%$ dos artigos encontraram relação positiva entre os dois constructos (Britner \& Pajares, 2006; Chan, 2008; Ciani, Summers, \& Easter, 2008; Gibbs \& Powell, 2012; Kurt, Duyar, \& Çalik, 2012; Lev \& Koslowsky, 2009; Parks, Solmon, \& Lee, 2007; Skaalvik \& Skaalvik, 2007; Viel-Ruma, Houchins, Jolivette, \& Benson, 2010; Ware \& Kitsantas, 2007; Zambo \& Zambo, 2008).

Descobriu-se que a eficácia coletiva prevê alguns aspectos efetivamente específicos da autoeficácia docente em situações distintas, como, por exemplo, a autoeficácia para a gestão de sala de aula entre os membros da equipe. Nesse caso específico, entende-se que o senso elevado de eficácia pessoal pode influenciar a maneira como professores administram as atividades em sala de aula, conferindo-lhes maior domínio de situações relacionadas a disciplina escolar, estratégias de ensino, controle de situações adversas, entre outras. Isso significa que grupos docentes com crenças elevadas de eficácia coletiva tendem a ser, individualmente, mais eficientes na gestão de sala de aula (Lev \& Koslowsky, 2009).

Kurz e Knight (2004), em suas investigações, constataram que a correlação entre eficácia coletiva e autoeficácia foi positiva e moderada $(\mathrm{r}=0,23, \mathrm{p}<0,05)$, embora entendam que é razoável dizer que ambos osconstructos são interdependente. Esses resultados indi- cam que forças distintas no ambiente de trabalho moldam a eficácia coletiva e a autoeficácia. Percepções diferentes dos professores a respeito das suas próprias crenças sobre a capacidade de gerar mudanças significativas e as suas crenças na capacidade dos seus colegas, podem contribuir para provocar uma diferença entre as crenças coletivas e pessoais de eficácia docente.

Skaalvik e Skaalvik, (2007) examinaram a relação causal entre autoeficácia e eficácia coletiva em 244 professores do Ensino Fundamental e do Ensino Médio da Noruega. A análise fatorial realizada nos dados indicou que a eficácia coletiva foi positiva e fortemente relacionada à autoeficácia docente $(\beta=.64, \mathrm{p}<.05)$. Ressalta-se que neste estudo foram detectadas relações causais recíprocas entre as variáveis.

Os estudos têm indicado que a eficácia coletiva docente e autoeficácia de professores são constructos distintos, e que as quatro fontes de informação (experiências diretas, experiências vicárias, persuasão social e estados afetivos e fisiológicos) que geram a autoeficácia também constituem as bases das crenças coletivas.

\section{Desempenho de alunos}

A variável desempenho dos alunos totalizou 36\% dos artigos encontrados. Os resultados indicaram que $100 \%$ dos estudos identificaram uma relação positiva entre eficácia coletiva docente e desempenho dos alunos (Cybulski, Hoy, \& Sweetland, 2005; Goddard, 2001,2002, 2006; Goddard, Logerfo, \& Hoy, 2004; Goddard, Hoy, \& Hoy, 2000; Hoy, Sweetland, \& Smith, 2002; Kurt et al., 2012; Kurz \& Knight, 2004; Moolenaar, Sleegers, \& Daly, 2012; Putney \& Broughton, 2011).

As pesquisas apontam que a eficácia coletiva docente pode influenciar o desempenho dos alunos. No entanto, em alguns casos, essas crenças coletivas podem explicar apenas de forma moderada a variação do desempenho, pois outros fatores podem influenciar o processo de ensino-aprendizagem, tais como os aspectos socioeconômicos da escola (Cybulski, Hoy, \& Sweetland, 2005; Goddard, 2001; Goddard, Logerfo, \& Hoy, 2004; Hoy, Sweetland, \& Smith, 2002; Moolenaar, Sleegers, \& Daly, 2012).

Goddard (2001) investigou a variabilidade da percepção dos professores em relação às crenças de eficácia coletiva docente, analisando também a relação entre esta variável e o desempenho dos alunos. Participaram da pesquisa 452 professores de 91 escolas do ensino 
fundamental nos Estados Unidos. Os resultados indicaram que desempenho acadêmico apresentou correlação positiva com eficácia coletiva $(\mathrm{r}=.77, \mathrm{p}<.001)$.

Hoy et al., (2002) buscaram construir um modelo teórico para explicar o desempenho dos alunos. Percebeu-se que a eficácia coletiva docente é uma variávelchave e também teve correlação positiva com o desempenho dos alunos $(\mathrm{r}=.65, \mathrm{p}<.001)$. $\mathrm{O}$ estudo foi realizado em 97 escolas de ensino médio em Ohion, nos Estados Unidos. Sendo assim, notou-se, nesta pesquisa, que a eficácia coletiva é uma das variáveis mais importantes para explicar o desempenho acadêmico dos alunos. Entende-se que o forte senso de eficácia coletiva pode levar os professores a ser mais persistentes em seus esforços para conseguir melhores resultados no processo de ensino-aprendizagem, superando possíveis dificuldades. Isso pode ser um fator relevante, capaz de influenciar diretamente as realizações acadêmicas dos alunos.

Na pesquisa de Moolenaar et al. (2012), um achado interessante revelou que as crenças de eficácia coletivas são preditoras das realizações acadêmicas dos alunos em linguagem, mas não para resultados escolares relacionados à matemática. Embora inesperado, esse dado se justificou, segundo os autores, por questões sociais, econômicas e políticas que envolvem o sistema educacional do local de coleta dos dados. Nesse caso específico havia muito mais investimento político e financeiro direcionado para as práticas de leitura do que para o ensino de matemática. Percebe-se, assim, que a estrutura dos sistemas educacionais também pode explicar, em parte, a variação do desempenho acadêmico dos alunos.

\section{Situação socioeconômica (SES) da escola}

A relação entre eficácia coletiva docente e situação socioeconômica (SES) da escola foi investigada em $20 \%$ dos artigos. Todos os resultados indicaram que as variáveis estão significativamente relacionadas (Adams \& Forsyth, 2006; Cybulski et al., 2005; Gibbs \& Powell, 2012; Hoy et al., 2002; Kurz \& Knight, 2004; McCoach \& Colbert, 2010).

Hoy et al. (2002) realizaram pesquisa com 97 escolas de ensino médio para construir um modelo teórico visando explicar o desempenho escolar. A eficácia coletiva foi a variável-chave do estudo em um sistema teórico que envolvia outras variáveis, entre elas a situação socioeconômica. Os pesquisadores encontraram relação causal entre as variáveis. A situação socioeconômica da escolateve efeito positivo sobre a eficácia coletiva ( $\beta=$ $.25, \mathrm{p}<.05)$. Isso sugere que, quanto melhor for a situ- ação socioeconômica de uma escola, mais elevadas serão as crenças coletivas de eficácia.

Goddard et al., (2004) realizaram um estudo para examinar a relação entre eficácia coletiva e outras variáveis de contexto escolar, numa amostra de 96 escolas de ensino médio. A situação socioeconômica das escolas foi positivamente correlacionada com as crenças coletivas dos professores $(r=0,31, p<0,05)$. Sugerese, neste estudo, que os professores tendem a ter um senso mais forte de capacidade de grupo quando instruem alunos pertencentes a classes sociais mais altas e com melhores condições de vida.

Adams e Forsyth (2006) investigaram a influência das variáveis contextuais sobre as crenças de eficácia coletiva. A pesquisa foi realizada com a participação de 595 professores de 79 escolas de ensino fundamental e médio da cidade de Oklahoma, nos Estados Unidos. Notou-se que a situação socioeconômica e a eficácia coletiva foram inversamente proporcionais $(r=-.60, p$ $<=.001$ ). Altas porcentagens de alunos elegíveis para receber a ajuda financeira federal no almoço, em virtude da situação de pobreza, estão associadas aos baixos níveis de eficácia coletiva de professores. Os resultados indicaram ainda que a situação socioeconômica é preditora da eficácia coletiva docente $(\beta=-.23, \mathrm{p}<.01)$. Isso sugere que escolas com maior nível de pobreza estão predispostas a condições que podem impedir aconstituição das crenças de eficácia coletiva docente. Essas condições relacionam-se às dificuldades próprias do contexto de pobreza, as quais podem criar limitações e problemas no processo de ensino-aprendizagem, afetando a percepção de eficácia em relação à capacidade do corpo docente.

Os estudos indicam que desvantagens sociodemográficas e socioeconômicas podem influenciar no rebaixamento ou na elevação das crenças de eficácia coletiva docente. No entanto, acredita-se que diante de crenças coletivas elevadas os efeitos negativos dos aspectos sociodemográficos podem ser reduzidos (Parker, Hannah, Topping, 2006). Por isso, é importante investir na elevação das crenças, uma vez que, provavelmente, é mais fácil mudar o nível das crenças de eficácia coletiva do que os aspectos sociodemográficos, pois existem implicações políticas e estruturais envolvidas nesse processo (Parker, Hannah, Topping, 2006). É preciso considerar que provavelmente, professores com crenças coletivas elevadas podem conseguir um bom desempenho no processo de ensino-aprendizagem, a despeito das desvantagens socioeconômicas das escolas (Cybulski et al., 2005). 


\section{Satisfação no trabalho}

A variável satisfação no trabalho foi investigada em conjunto com a eficácia coletiva docente em $6 \%$ dos estudos (Klassen \& Chiu, 2010; Viel-Ruma, Houchins, Jolivette, \& Benson, 2010). Em todos os casos houve relação positiva entre as variáveis.

Klassen et al. (2010) examinaram a relação entre satisfação no trabalho e eficácia coletiva docente. Perceberam que contextos culturais apontam, especificamente, diferenças significantes nos papéis desempenhados pela eficácia coletiva e suas ligações com a satisfação no trabalho. Esse estudo foi um dos primeiros a examinar as crenças coletivas de professores usando uma estrutura intercultural, investigando dados de países diferentes (dois países da América do Norte Canadá e Estados Unidos - , e um país do Leste Asiático - Coréia do Sul). Concluiu-se que as pesquisas sobre motivação docente só terão valor prático se seus achados forem entendidos como limitados por uma série de fatores culturais, que devem ser considerados e compreendidos.

No estudo de Klassen et al. (2010), a eficácia coletiva foi positivamente relacionada à satisfação no trabalho docente, mesmo considerando a realidade de diferentes países, a saber: Canadá $(\mathrm{r}=.42, \mathrm{p}<.01)$, Estados Unidos $(\mathrm{r}=.37, \mathrm{p}<.01)$; Coreia do Sul $(\mathrm{r}=.48$, $\mathrm{p}<.01)$. Descobriu-se, por meio de análise de trilha, que não houve diferença significativa na contribuição da eficácia coletiva para a satisfação no trabalho docente, mesmo considerando as especificidades culturaisda amostra envolvida. A análise de trilha permitiu identificar a capacidade de previsão (causa e efeito) de uma variável em relação a outra. Para avaliar a eficácia coletiva, os pesquisadores utilizaram duas dimensões que foram investigadas pela análise de trilha: estratégias de ensino (.16/norte-americanos e .15/sul-coreanos) e disciplina escolar (. 26/ norte-americanos e $.25 /$ sul-coreanos). Em ambas as dimensões, a eficácia coletiva foi capaz de explicar ( $<<.01, \mathrm{p}<.001$, respectivamente) a satisfação no trabalho docente sem diferenças significativas entre professores norte-americanos e sul-coreanos.

Sugere-se que, no caso dos professores sul-coreanos, a eficácia coletiva se desenvolve porque a relação entre as variáveis é o resultado de uma ênfase cultural mais forte em evitar conflitos e melhorar a harmonia do grupo. Nesse estudo, no entanto, os professores norteamericanos demonstraram, surpreendentemente, possuir um senso comparativamente elevado de eficácia coletiva, mesmo sendo de uma cultura reconhecidamente individualista. Considera-se que esse senso de grupo pode ter se formado para atender a interesses individuais. Sendo assim, acredita-se que a eficácia coletiva e a satisfação no trabalho se desenvolvem de formas diferentes nessas culturas. Para os professores norte-americanos se sentirem satisfeitos com o trabalho é necessário fornecer apoio administrativo, condições para controlar o ambiente de ensino e oportunidades para influenciar as políticas educacionais, pois exigem mais autonomia individual para a realização de suas funções. Isso consequentemente, gera um senso de competência de grupo, elevando a eficácia coletiva. Para os professores sul-coreanos, no entanto, a satisfação no trabalho se desenvolve quando há, primeiramente, um forte senso de eficácia coletiva, que se constitui por meio de um clima organizacional harmônico e não conflituoso (Klassen, Usher, et al., 2010).

Notou-se que a eficácia coletiva e a satisfação no trabalho são variáveis que podem se influenciar mutuamente. Os professores que estão muito satisfeitos com sua experiência de trabalho relatam níveis mais elevados de crenças de eficácia coletiva (Bandura, 1997, 1998; Klassen et al., 2010; Stephanou, 2013). Os resultados sugerem que o investimento na melhoria das crenças de eficácia coletiva poderia elevar o nível de satisfação no trabalho e, consequentemente, melhorar o desempenho do professor.

\section{Tempo de serviço}

A relação entre as variáveis eficácia coletiva docente e tempo de serviço foi investigada em $6 \%$ dos casos. Nos dois artigos que analisaram essa relação os seguintes resultados foram encontrados: relação negativa (Skaalvik \& Skaalvik, 2007) e relação positiva (Chan, 2008).

Skaalvik e Skaalvik (2007), no estudo com professores de ensino fundamental e médio, também investigaram a relação causal entre tempo de serviço e eficácia coletiva. Os resultados indicaram que tempo de serviço tem um efeito negativo $(\beta=.-23, \mathrm{p}<.05)$ sobre a eficácia coletiva. Percebe-se, nesse estudo, que, quanto mais anos de serviço o professor tem, menores são as crenças coletivas dele.

Chan (2008) avaliou, entre outras variáveis, a eficácia coletiva em 273 professores de Hong Kong. Notouse que tempo de serviço foi correlacionado positivamente $(\mathrm{r}=.17, \mathrm{p}<.01)$ com eficácia coletiva docente, embora a intensidade da correlação tenha sido fraca. Sugere-se, nesse caso, que os anos de experiência de ensino influenciam as crenças de eficácia: quanto mais tempo de serviço os docentes tiverem, mais elevadas serão suas crenças. 


\section{Estresse no trabalho}

A relação entre estresse no trabalho e eficácia coletiva docente foi investigada em $6 \%$ dos artigos e todos eles encontram relação negativa entre as variáveis(Klassen et al., 2010; Klassen, 2010). A literatura da área tem indicado que professores expostos a experiências que provocam emoções negativas tendem a sofrer um rebaixamento nas crenças de eficácia coletiva. As consequências do estresse podem ser graves provocando o desencadeamento de burnout, depressão, mau desempenho e, por fim, a decisão de desistir da função. $\mathrm{O}$ estresse docente pode ser evitado quando a escola fornece condições para que o professor desempenhe seu trabalho com satisfação.

Klassen (2010) explorou a relação entre estresse no trabalho e eficácia coletiva docente com a participação de 951 professores de escolas de ensino fundamental e médio, no Canadá. Percebeu-se que o estresse foi negativamente correlacionado à eficácia coletiva $(\mathrm{r}=.-12, \mathrm{p}$ $<=.01$ ), sugerindo que, quanto maior for o nível de estresse no trabalho, menores serão as crenças de eficácia coletiva.

\section{CONSIDERAÇÕES FINAIS}

A presente revisão sistemática possui algumas limitações decorrentes da escolha dos critérios de inclusão. Neste estudo, as buscas dos artigos foram realizadas apenas na base de dados do Portal de Periódicos - Capes (Brasil), que possui grande acervo de pesquisas tanto em nível nacional quanto internacional. Sugere-se que futuras pesquisas ampliem suas buscas utilizando outras bases de dados.

A partir dos resultados da presente revisão sistemática foi possível identificar, nessa área de estudo, algumas lacunas que necessitam de maior atenção por parte dos pesquisadores, visando a uma melhor compreensão acerca do constructo investigado. Dentre essas lacunas, destaca-se a relação entre eficácia coletiva docente e desempenho de alunos. Apesar de alguns (33\%) dos estudos analisados terem pesquisado essa relação, percebe-se a necessidade de aprofundamento, explorando esses constructos em contextos diversos, como, por exemplo, em escolas com grande desvantagem socioeconômica, com alta pobreza, bem como em escolas de ensino médio. As pesquisas mais frequentemente têm sido desenvolvidas em escolas de ensino fundamental.

Entende-se que ainda são poucos os estudos que se dedicam a investigar a constituição das crenças de eficácia coletiva docente. Pesquisas futuras poderão examinar a relação entre diferentes variáveis contextuais e crenças coletivas de eficácia. São necessárias mais investigações sobre a relação entre variáveis sociodemográficas, culturais e econômicas e as crenças coletivas dos professores para explicar o impacto dessas variáveis no processo de ensino-aprendizagem.

A identificação das características metodológicas indica, também, que é importante ampliar os estudos com abordagem qualitativa e de natureza longitudinal. Pesquisas que abordam a natureza qualitativa dos dados poderão contribuir significativamente com a compreensão dos dados produzidos pelos pesquisadores do tema.

Outro aspecto que necessita de atenção éa relação entre as crenças pessoais e as crenças coletivas de eficácia docente. Mais estudos são necessários para verificar o nível de interdependência entre esses constructos, bem como a forma como atuam na rotina escolar.

Os resultados da presente revisão sistemática indicaram, ainda, que é preciso desenvolver estudos para explorar outras lacunas, tais como: análise das crenças de professores de ensino superior, o impacto da formação de professores nas crenças coletivas; implicações do burnout nas crenças de eficácia docente de professores e variáveis de estresse, satisfação no trabalho e redes sociais.

Em síntese, esta revisão permitiu perceber que há uma escassez de artigos relacionados ao tema eficácia coletiva de professores. Se, por um lado isso tornou as buscas difíceis, por outro, indicou a necessidade de mais pesquisas para descrever esse fenômeno e suas implicações, uma vez que o tema possui um papel reconhecidamente importante nos desempenhos do professor e dos alunos. Nesse sentido, com a presente revisão, pretende-se despertar o interesse da comunidade científica em geral, de modo que mais estudos sobre esse tema sejam desenvolvidos.

\section{REFERÊNCIAS}

Adams, C. M., \& Forsyth, P. B. (2006). Proximate sources of collective teacher efficacy. Journal of Educational Administration, 44(6),
doi:10.1108/09578230610704828

Bandura, A. (1986). Social foundations of thought and action: A social cognitive theory. Book. Prentice-Hall, Inc. Retrieved from http://books.google.de/books?hl=de\&lr=\&id=rcnJB7Wkr9YC\&oi=fnd\&pg=PA94 $\& \mathrm{dq}=$ the + social + foundations + of + thought + and + action\&ots=DYNNnRPwhK\&sig=ajTjjUIM2PPvAz2Qvjm4Wch-gEc

Bandura, A. (1993). Perceived SelfEfficacy in Cognitive Development and Functioning.pdf. Educational Psychologist, 28(2), 117-148. 
Bandura, A. (1994). Self-Efficacy. Encyclopedia of Human Behavio, 4(1994), 71-81.

Bandura, A. (1997). Self-efficacy: The exercise of control. New York: W. H. Freeman. Retrieved from http://books.google.com/books?id=eJ-PN9g o-EC\&print$\mathrm{sec}=$ frontcover $\& \mathrm{dq}=$ bandura $+\mathrm{isbn}: \overline{9} 780716728504 \& \mathrm{hl}=\mathrm{e}$ n\&ei=HAwYTbKsLpTmsQPp8cCPCg\&sa=X\&oi=book_result\&ct=result\&resnum $=1 \&$ ved $=0$ CCMQ6AEwAA\# $=$ onepage \&q\&f=false

Bandura, A. (1998). Health promotion from the perspective of social cognitive theory. Psychology \& Health, 13(4), 623-649. doi:10.1080/08870449808407422

Bandura, A. (2000). Exercise of Human Agency Through Collective Efficacy. Current Directions in Psychological Science, 9(3), 75-78. doi:10.1111/1467-8721.00064

Bandura, A., Lindzey, G., \& Runyan, W. M. (2007). Albert Bandura. In A history of psychology in autobiography, Vol. IX. (pp. 43-75). American Psychological Association. Retrieved from 10.1037/11571-002\nhttp://search.ebscohost.com/login.aspx?direct $=$ true $\& \mathrm{db}=$ psyh\&AN =2007-00058-002\&amp;lang $=$ fr\&site $=$ ehost-live

Britner, S. L., \& Pajares, F. (2006). Sources of science selfefficacy beliefs of middle school students. Journal of Research in Science Teaching, 43(5), 485-499. doi:10.1002/tea.20131

Bzuneck, J. A., \& Guimarães, S. É. R. (2009, November 6). Eficácia coletiva dos professores e implicações para o contexto educacional brasileiro Teacher's collective efficacy and implications for brazilian' education. ETD Educação Temática Digital. Retrieved from http://www.fae.unicamp.br/revista/index.php/etd/article/view/2052

Chan, D. W. (2008). General, collective, and domain-specific teacher self-efficacy among Chinese prospective and inservice teachers in Hong Kong. Teaching and Teacher Education, 24(4), 1057-1069. doi:10.1016/j.tate. 2007.11 .010

Ciani, K. D., Summers, J. J., \& Easter, M. a. (2008). A “topdown" analysis of high school teacher motivation. Contemporary Educational Psychology, 33(4), 533-560. doi:10.1016/j.cedpsych.2007.04.002

Cybulski, T. G., Hoy, W. K., \& Sweetland, S. R. (2005). The roles of collective efficacy of teachers and fiscal efficiency in student achievement. Journal of Educational Administration, 43(5), doi:10.1108/09578230510615224

Dancey, C. P., \& Reidy, J. (2006). Estatística sem matemática para psicologia. Artmed (p. 608). Artmede.

Dantas, M. A. (2000). Eficacia coletiva de professores: análise se esalas internacionais de avaliação, 11(2), $181-190$.

Gibbs, S., \& Powell, B. (2012). Teacher efficacy and pupil behaviour: the structure of teachers' individual and collective beliefs and their relationship with numbers of pupils excluded from school. The British Journal of Educational Psychology, 82(Pt 4), 564-84. doi:10.1111/j.2044-8279.2011.02046.

Goddard, R. (2002). A Theoretical and Empirical Analysis of the Measurement of Collective Efficacy: The Development of a Short Form. Educational and Psychological Measurement, 62(1), 97-110. doi:10.1177/0013164402062001007
Goddard, R. D. (2001). Collective efficacy: A neglected construct in the study of schools and student achievement. Journal of Educational Psychology, 93(3), 467-476. doi:10.1037//0022-0663.93.3.467

Goddard, R. D. (2006). The Influence of School Social Composition on Teachers' Collective Efficacy Beliefs. Educational Administration Quarterly, 42(2), 216-235. doi:10.1177/0013161X05285984

Goddard, R. D., Hoy, W. K., \& Hoy, a. W. (2000). Collective Teacher Efficacy: Its Meaning, Measure, and Impact on Student Achievement. American Educational Research Journal, 37(2), 479-507. doi:10.3102/00028312037002479

Goddard, R. D., Logerfo, L., \& Hoy, W. K. (2004). High School Accountability: the Role of Perceived Collective Efficacy. Educational Policy, 18(3), 403-425. doi:10.1177/0895904804265066

Hoy, W. K., Sweetland, S. R., \& Smith, P. a. (2002). Toward an Organizational Model of Achievement in High Schools: The Significance of Collective Efficacy. Educational Administration Quarterly, 38(1), 77-93. doi:10.1177/0013161X02381004

Klassen, R. M. (2010). Teacher Stress: The Mediating Role of Collective Efficacy Beliefs. The Journal of Educational Research, 103(5), 342-350. doi:10.1080/00220670903383069

Klassen, R. M., \& Chiu, M. M. (2010). Effects on teachers' self-efficacy and job satisfaction: Teacher gender, years of experience, and job stress. Journal of Educational Psychology, 102(3), 741-756. doi:10.1037/a0019237

Klassen, R. M., Tze, V. M. C., Betts, S. M., \& Gordon, K. a. (2010). Teacher Efficacy Research 1998-2009: Signs of Progress or Unfulfilled Promise? Educational Psychology Review, 23(1), 21-43. doi:10.1007/s10648-010-9141-8

Klassen, R. M., Usher, E. L., \& Bong, M. (2010). Teachers' Collective Efficacy, Job Satisfaction, and Job Stress in Cross-Cultural Context. The Journal of Experimental Education, 78(4), 464-486. doi:10.1080/00220970903292975

Kurt, T., Duyar, I., \& Çalik, T. (2012). Are we legitimate yet?: A closer look at the casual relationship mechanisms among principal leadership, teacher self-efficacy and collective efficacy. Journal of Management Development, 31(1), 71-86. doi:10.1108/02621711211191014

Kurz, T. B., \& Knight, S. L. (2004). An Exploration of the Relationship Among Teacher Efficacy, Collective Teacher Efficacy, and Goal Consensus. Learning Environments Research, 7(2), 111-128. doi:10.1023/B:LERI.0000037198.37750.0e

Lev, S., \& Koslowsky, M. (2009). Moderating the collective and self-efficacy relationship. Journal of Educational Administration, 47(4), 452-462. doi:10.1108/09578230910967437

McCoach, D. B., \& Colbert, R. D. (2010). Factors Underlying the Collective Teacher Efficacy Scale and Their Mediating Role in the Effect of Socioeconomic Status on Academic Achievement at the School Level. Measurement and Evaluation in Counseling and Development, 43(1), 31-47. doi:10.1177/0748175610362368

McCullough Chavis, A. (2011). Social Learning Theory and Behavioral Therapy: Considering Human Behaviors within the Social and Cultural Context of Individuals and 
Families. Social Work in Public Health, 26(5), 471-81. doi:10.1080/19371918.2011.591629

Minayo, M. C. de S., \& Sanches, O. (1993). Quantitativoqualitativo: oposição ou complementaridade? Cadernos de Saúde Pública. doi:10.1590/S0102311X1993000300002

Moolenaar, N. M., Sleegers, P. J. C., \& Daly, A. J. (2012). Teaming up: Linking collaboration networks, collective efficacy, and student achievement. Teaching and Teacher Education, 28(2), 251-262. doi:10.1016/j.tate.2011.10.001

Parker, K.; Hannah, E.; Topping, J. K. . (2006). Collective teacher efficacy, pupil attainment and socio-economic status in primary school(R). Improving Schools, 9(2), 111-129. doi:10.1177/1365480206064965

Parks, M., Solmon, M., \& Lee, A. (2007). Understanding Classroom Teachers' Perceptions of Integrating Physical Activity: A Collective Efficacy Perspective. Journal of Research in Childhood Education, 21(3), 316-328. doi:10.1080/02568540709594597

Putney, L. G., \& Broughton, S. H. (2011). Developing Collective Classroom Efficacy: The Teacher's Role as Community Organizer. Journal of Teacher Education, 62(1), 93-105. doi:10.1177/0022487110381760

Skaalvik, E. M., \& Skaalvik, S. (2007). Dimensions of teacher self-efficacy and relations with strain factors, perceived collective teacher efficacy, and teacher burnout. Journal of Educational Psychology, 99(3), 611-625. doi:10.1037/0022-0663.99.3.611

Stephanou, G. (2013a). The Role of Teachers' Self- and Collective-Efficacy Beliefs on Their Job Satisfaction and Experienced Emotions in School. Psychology, 04(03), 268-278. doi:10.4236/psych.2013.43A040

Stephanou, G. (2013b). The Role of Teachers' Self- and Collective-Efficacy Beliefs on Their Job Satisfaction and Experienced Emotions in School. Psychology, 04(03), 268-278. doi:10.4236/psych.2013.43A040

Tschannen-Moran, M., Hoy, A. W., \& Hoy, W. K. (1998). Teacher Efficacy: Its Meaning and Measure. Review of Educational Research, 68(2), 202-248. doi:10.3102/00346543068002202
Tschannen-moran, M., \& Mcmaster, P. (2013). Sources of Self-Efficacy: Four Professional Development Formats and Their Relationship to Self-Efficacy and Implementation of a New Teaching Strategy, 110(2), 228-245.

Viel-Ruma, K., Houchins, D., Jolivette, K., \& Benson, G. (2010a). Efficacy Beliefs of Special Educators: The Relationships Among Collective Efficacy, Teacher Self-Efficacy, and Job Satisfaction. Teacher Education and Special Education: The Journal of the Teacher Education Division of the Council for Exceptional Children, 33(3), 225-233. doi:10.1177/0888406409360129

Viel-Ruma, K., Houchins, D., Jolivette, K., \& Benson, G. (2010b). Efficacy Beliefs of Special Educators: The Relationships Among Collective Efficacy, Teacher Self-Efficacy, and Job Satisfaction. Teacher Education and Special Education: The Journal of the Teacher Education Division of the Council for Exceptional Children, 33(3), 225-233. doi:10.1177/0888406409360129

Ware, H., \& Kitsantas, A. (2007). Teacher and Collective Efficacy Beliefs as Predictors of Professional Commitment. The Journal of Educational Research, 100(5), 303-310. doi:10.3200/JOER.100.5.303-310

Zambo, D., \& Zambo, B. R. (2008). The Impact of Professional Development in Mathematics on Teachers ' Individual and Collective Efficacy: The Stigma of Underperforming. Teacher Education Quarterly, 35(1), 159-168.
Recebido em: 07/04/2014 Primeira decisao editorial em: 05/11/2014

Aceito em: 16/10/2015 